\title{
Las diferencias que crea el lugar. Una mirada crítica a la hegemonía angloamericana en geografía
}

\section{Maria-Dolors Garcia-Ramon}

Universitat Autònoma de Barcelona. Departament de Geografia mariadolors.garcia.ramon@uab.es

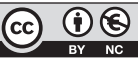

Recepción: julio de 2011

Aceptación: noviembre de 2011

\section{Resumen}

Hoy, quizás más que nunca en la historia de nuestra disciplina, se observa una indisputada hegemonía internacional de una única geografía, la angloamericana. Ésta establece las pautas del debate intelectual que tiene lugar en distintas partes del mundo, en gran parte debido a la preponderancia del inglés. Pero esta hegemonía lingüística es una forma de poder que, mientras dignifica ciertas tradiciones académicas, desautoriza otras. En este artículo me referiré, en primer lugar, a la situación de la geografía internacional una década después de un cierto debate sobre ello en los foros internacionales de geografía crítica y, en segundo lugar, al caso de la geografía feminista, que no es ninguna excepción en este proceso.

Palabras clave: geografía; hegemonía angloamericana; geografía feminista; conocimiento situado.

Resum. Les diferències que crea el lloc. Una mirada critica a l'hegemonia angloamericana en geografía

Avui, potser més que mai a la història de la nostra disciplina, s'observa una indisputada hegemonia internacional d'una única geografia, l'angloamericana. Aquesta estableix les pautes del debat intel.lectual que té lloc a diverses parts del món, en gran part a causa de l'hegemonia de l'anglès. Però aquesta preponderància lingüística és una forma de poder que, mentre dignifica certes tradicions acadèmiques, en desautoritza unes altres. En aquest article em referiré, en primer lloc, a la situació de la geografia internacional una dècada després d'un cert debat sobre el tema en els fòrums internacionals de geografia crítica i, en segon lloc, al cas de la geografia feminista, que no és cap excepció en aquest procés.

Paraules clau: geografia; hegemonia angloamericana; geografia feminista; coneixement situat.

Résumé. Les differences créés par le lieu. Un regard critique à l'hégémonie anglo-américaine en géographie

Aujourd'hui, peut-être plus que jamais dans l'histoire de notre discipline, une unique géographie, celle de la géographie anglo-américaine, est devenu d'une hégémonie internationale incontestée. Elle détermine les directives du débat intellectuel qui a lieu dans 
les différentes parties du monde, principalement à l'occasion de l'hégémonie de l'anglais. Mais cette hégémonie linguistique est une forme de pouvoir que si bien dignifie certaines traditions académiques aussi en discrédite d'autres. Dans ce article, je vais me référer d'abord à la situation de la géographie internationale d'après une décennie dans lequel ont prévalu quelques débats à ce sujet dans les forums internacionaux de géographie critique et, tout suite, à la question de la géographie féministe qui n'est pas une exception.

Mots clé: géographie; hégémonie anglo-américaine; la géographie féministe; savoir situé.

Abstract. The differences that place makes. A critical view on Anglo-American hegemony in geography

Today, perhaps more than ever in the history of our discipline, there is an undisputed international hegemony of a single geography: the Anglo-American. This has set the patterns of intellectual debate taking place in different parts of the world, largely due to the hegemony of English. However, this linguistic hegemony is a form of power that dignifies certain academic traditions, while disempowering others. This article first refers to the situation of international geography a decade after a debate on this issue was launched in international forums on critical geography and, secondly, the case of feminist geography, which is not an exception in this process.

Keywords: geography; Anglo-American hegemony; feminist geography; situated knowledge.

\section{Sumario}

La indisputada hegemonía de la geografía angloamericana

Las diferencias que crea el lugar.

Algunos datos numéricos sobre la geografía del género
Conocimientos «situados»

en geografia feminista

Mirando al futuro y produciendo conocimientos «situados»

Referencias bibliográficas

Durante la última década, y en algunos foros internacionales de geografía crítica, se ha debatido sobre la hegemonía angloamericana respecto a la producción del conocimiento en geografía y el estatus del inglés como lingua franca de la comunicación académica. A nivel personal, hace mucho tiempo que me preocupa el tema, quizás porque lo he experimentado y sufrido desde muy joven — desde que fui a Berkeley a hacer un máster en geografía en 1970 - , y porque mi trayectoria académica ha estado muy expuesta a la geografía anglosajona. También he de decir desde el principio que mi perspectiva no es anglocéntrica, pero, por supuesto, podría ser y es eurocéntrica, sin embargo, los países mediterráneos están en la periferia de Europa, una periferia que no ha sido «inventada» (Best, 2009) —ahora incluso se habla de los PIGS (cerdos) de la Unión Europea, jugando con las iniciales de Portugal, Irlanda, Grecia y España (Spain). Mi experiencia, pues, no está de ninguna manera compenetrada con las decisiones que se toman en los centros del 
poder ni en los centros de la geografía «internacional». Además, en Cataluña (con una sociedad civil anclada sobre una fuerte identidad colectiva y sobre una lengua propia, el catalán), tenemos una experiencia de trabajo y diálogo diario con los geógrafos españoles y una experiencia cotidiana de relacionarnos y de hablar en dos lenguas (catalán y castellano/español) que se encuentran en una posición de poder asimétrica. Por lo tanto, los problemas de lengua y poder y su relación asimétrica con el "Otro» los he vivido a diario y es lo que me interesa discutir aquí. A nivel internacional, está claro que la consolidación de la hegemonía del inglés como lengua global ha situado el discurso geográfico en un lugar de poder privilegiado dentro de la comunidad académica internacional de geógrafos y ello tiene numerosas consecuencias. En este artículo me referiré, en primer lugar, a la situación de la geografía internacional y, después, me centraré con más detalle en el caso de la geografía feminista, que no es ninguna excepción.

El tema de la hegemonía de la geografía anglosajona se ha venido discutiendo en la última década en algunos foros internacionales (sobre todo en los de geografía crítica y también en los de género) (Short et al., 2001; Braun, 2003; Minca, 2003; Rodríguez-Pose, 2004; Albet y Benach, 2005; Paasi, 2005). Por ejemplo: en la Conferencia Internacional de Geografía Crítica, que tuvo lugar en Hungría en el año 2002, los dos temas que dominaron las discusiones, tanto dentro como fuera de las conferencias, fueron la hegemonía angloamericana en la geografía internacional y el estatus del inglés como lingua franca en la academia. Algo parecido sucedió en muchas de las sesiones de la Conferencia Internacional de Geografía Crítica en México DF, en 2005. Poco después de estas conferencias, se publicaron algunos artículos (Kitchin, 2003; Garcia-Ramon, 2003, 2004; Vaiou, 2004; Timar, 2004; Rodríguez-Pose, 2006), pero en los últimos años, y con alguna honrosa excepción (Peake, 2011), parece que el tema no ha estado tan de moda en el mundo anglófono (las modas pasan muy deprisa en la geografía anglosajona), aunque es interesante el debate publicado recientemente en Area protagonizado por geógrafos belgas (Derudder, 2011; Meeus et al., 2011). Lo que es cierto es que, si bien se ha escrito sobre el tema, es muy poco lo que se ha hecho y la situación no ha cambiado, si es que no ha empeorado.

\section{La indisputada hegemonía de la geografía angloamericana}

Hoy, quizás más que nunca en la historia de nuestra disciplina, se observa una indisputada hegemonía internacional de una única geografía, la angloamericana. Ésta establece las pautas del debate intelectual que tiene lugar en distintas partes del mundo, en gran parte debido a la hegemonía del inglés. Pero esta hegemonía lingüística es una forma de poder que, mientras dignifica ciertas tradiciones académicas, desautoriza otras. Además, el lenguaje no sólo refleja el mundo externo, sino que también lo materializa o lo corporifica, pues el lenguaje es más que una herramienta comunicativa de intercambio de ideas; el lenguaje condiciona una manera de pensar y establece el 
marco en el que expresamos nuestras experiencias y percepciones. Por ello, el problema de las diferentes tradiciones académicas en geografía debe ser incorporado en nuestra discusión sobre las lenguas y la hegemonía de un pensamiento único. Es verdad que el discurso geográfico se ha globalizado, pero su globalización ha sido parcial y desigual. Quienes pueden hablar y escribir en inglés pueden contar con un público internacional, pero quienes hablan y escriben en otras lenguas tienen una difusión restringida no sólo a las conferencias internacionales, sino también a la mayor parte de las revistas de geografía contemporáneas más destacadas y conocidas. Nos guste o no, el acceso a estas publicaciones significa tener el poder real para establecer las pautas del debate intelectual en geografía en muchas zonas del mundo.

Parecería que las revistas consideradas «internacionales» serían el foro natural en que las diferentes comunidades internacionales de geógrafos pudieran comunicarse. Pero quizás el error sea identificar estas revistas como un foro de discusión internacional. Un estudio llevado a cabo por dos geógrafos españoles (Gutiérrez y López-Nieva, 2001) y publicado en Progress in Human Geography demuestra que las publicaciones denominadas «internacionales» en inglés no son realmente internacionales y que no han conseguido crear un foro global de discusión o, en realidad, quizás nunca se buscó que fuera este tipo de foro. El análisis se basa en una muestra de diecinueve revistas elegidas entre las treinta que aparecen bajo la categoría "Geografía» en el Social Sciences Citation Index. Si se toma en cuenta el lugar de trabajo de los autores, Estados Unidos e Inglaterra, representan casi las tres cuartas partes de todos los artículos publicados (73,4\%). Canadá y Australia le siguen de forma inmediata y la contribución de los países de habla no inglesa es extremadamente baja (por ejemplo: Francia y Alemania, con escuelas de geografía muy potentes desde hace mucho tiempo, aparecen con un valor simbólico bajo, de alrededor de un $0,5 \%$ ). Más aún, la mayor parte de los miembros de los consejos editoriales de estas publicaciones provienen de países de habla inglesa. Otros dos geógrafos europeos (uno italiano y otro danés) constatan esta misma situación, a pesar de la diferencia de años en su publicación (Aalbers y Rossi, 2007, 2009).

Además, estas revistas probablemente no son sentidas por muchos geógrafos como un terreno adecuado para el encuentro y el debate entre diversas tradiciones geográficas, pues existe una convicción bastante generalizada entre nuestros colegas no anglosajones (por ejemplo: italianos, franceses, portugueses, españoles, brasileños, argentinos, etc.) de que estas revistas hacen referencia casi exclusivamente a los últimos debates teóricos dentro de la geografía angloamericana y que no dan cabida a "Otras» tradiciones (Hancock, 1999). Este sentimiento ha sido reforzado por el hecho de que el sistema de evaluación (referees) de estas revistas es casi exclusivamente angloamericano. Además, me gustaría añadir que, en muchos países y para la mayoría de las disciplinas, cada vez más, el Citation Index (es decir las revistas incluídas en el ISI) se está convirtiendo en el punto de referencia para medir la calidad de las publicaciones y las consiguientes promociones académicas, y la geografía no es ninguna excepción en este aspecto (es cierto que, en los últimos años, 
el ISI ha incluido algunas revistas no anglófonas, pero son escasas y con índices de impacto muy bajos, debido al sistema de elaboración del ISI).

En definitiva, si un libro o un artículo no esta escrito en inglés (o traducido a esta lengua), no existe para la comunidad angloparlante. Es tan simple como esto. Dejadme poner un ejemplo que me toca muy de cerca, pues el autor es español. Recuerdo que, en Barcelona, estábamos ya familiarizados con la obra de Manuel Castells en español hacia el año 1969 y la usábamos en nuestras investigaciones en geografía urbana (así como también lo hacían algunos de nuestros colegas italianos y franceses), pero Castells simplemente no existía para nuestros colegas de habla inglesa. En 1977, ocho años después, de repente, muchos de nuestros colegas angloparlantes hacían profusas referencias al mismo y ello lo convirtió en el académico más citado en geografía urbana en los foros «internacionales». La explicación es muy simple: su famoso libro La question urbaine (1972) fue traducido del francés al inglés en 1977 (año en que Harvey estaba en París y conoció a Castells y a su obra).

Otro ejemplo significativo, esta vez en la geografía feminista internacional, es el caso del excelente manual publicado por el grupo WGSG (1997), Feminist Geographies: Exploration in diversity and difference. El subtítulo realmente aboga por la diversidad, la diferencia (conceptos muy queridos por la geografía del género) y, por lo tanto, por el conocimiento situado, siguiendo a Haraway (1995) y a Monk (1996). No obstante, el contenido se refiere tan solo a la geografía angloamericana, y la bibliografía, con la excepción de un par de referencias, sólo incluye las investigaciones publicadas y llevadas a cabo por la geografía anglófona. En cambio, se ignoran las investigaciones de geógrafas italianas, españolas, portuguesas, griegas y francesas, a pesar de que algunas de ellas publican en inglés. La geografía feminista (y, por descontado, las autoras del libro) reconoce que el conocimiento está situado, pero me gustaría añadir que el conocimiento no solo está situado dentro del mundo anglosajón. En mi opinión, un título más adecuado para este libro sería Feminist Geographies: Exploration in diversity and difference within the Angloxason word. Y este ejemplo se puede generalizar a los libros de texto en inglés de muchas otras materias de la geografía.

Aprender una lengua extranjera requiere tiempo y una gran dosis de humildad (es muy cómodo hablar en una lengua en la que no se cometen errores), pero este esfuerzo debe ser compartido por todo el mundo y no sólo por aquellos que no tienen al inglés como lengua materna. Como mujer, me agrada la comparación hecha por una geógrafa del Grupo de Geografía Crítica: «Si el inglés no es nuestra lengua materna, el esfuerzo realizado para seguir los debates de la geografía internacional es grande y mucha gente lo considera injusto. Lo mismo sucede con la sub-representación de mujeres en la disciplina; para una mujer que quiere seguir la "normalidad demográfica» de una vida familiar, competir por un buen trabajo significa un mayor esfuerzo que para un hombre que puede apoyarse en las normas sociales que le permiten contar con una compañera que acarreará con el mayor peso de las responsabilidades familiares. Tanto el hablar inglés como el ser hombre liberan más 
energía para el trabajo competitivo [...] estaría muy bien que todos encontráramos tiempo para consideraciones de carácter más humano, como ampliar nuestra comprensión de otras lenguas y asumir responsabilidades iguales en la vida familiar» (Critical Geographycal Forum Archives, 1998).

Algunas veces se han escuchado voces que objetan que el conocimiento de lenguas puede ser entendido también como un rasgo de elitismo (Critical Geographycal Forum Archives, 1998). Para quienes no tenemos el inglés como lengua materna, esta justificación del monolingüismo es más bien chocante - e incluso insultante- y revela hasta qué punto las ventajas de ser un angloparlante nativo puede considerarse una especie de don «natural».

Cabe señalar que la hegemonía anglosajona en geografía es muy compleja (Timar, 2004) y que, dentro mismo del ámbito anglófono, se da una graduación de hegemonías y unas geometrías variables del poder (Berg y Kearn, 1998; Samers y Sidaway, 2000). En la Conferencia Internacional de la UGI realizada en Brisbane (Australia), en 2006, en una sesión titulada «Antipodean Socio-spatial theory», se debatió brillantemente la preponderancia de la geografía norteamericana y la británica como centros de poder dentro de la geografía anglófona. En la conferencia plenaria del norteamericano Eric Shepard y los comentarios posteriores de los australianos (Pauline McGuirk y Phillip O'Neill), se llegó a denominar a estos dos países como las "Torres Gemelas» de la geografía internacional y se criticó la "periferización» de algunos países anglófonos como Australia y Nueva Zelanda. Pero esto no quita que estos países no dejen de estar algo más próximos que los no anglófonos al centro de poder, aunque no sea más que por el hecho de hablar inglés y, por lo tanto, dominar la lingua franca, un instrumento claro de preponderancia.

También es cierto que la internacionalización de la geografía no siempre refuerza la hegemonía angloamericana y muchas veces puede tener consecuencias muy positivas. Así pues, se ha constatado que las corrientes de geografía crítica se han introducido en Alemania gracias a la internacionalización de la geografía alemana (Belina et al., 2009). Y lo mismo podríamos decir de la introducción de la geografía feminista y del género en numerosos países donde es cierto que la geografía británica y americana ha desempeñado un papel esencial (Garcia-Ramon y Monk, 2007). Frente a esta situación, creo que tenemos que plantear algunas soluciones. Deberíamos buscar foros alternativos para la discusión internacional en geografía, especialmente la feminista.

\section{Las diferencias que crea el lugar. Algunos datos numéricos sobre la geografía del género}

A pesar de que la geografía y la teoría feminista han sido pioneras en defender la diferencia y la diversidad, en la realidad diaria se olvida con demasiada frecuencia. Por lo general, se acepta que los temas y las metodologías de estudio de la geografía anglófona son la norma y el punto de referencia para la geografía feminista mundial. Es cierto que en muchos países debemos el ini- 
cio y los primeros desarrollos de la geografía del género a su influencia, pero ello no supone que la evolución posterior se tenga que homologar con la de la anglosajona, ya que las tradiciones geográficas locales condicionan mucho su desarrollo. En un estudio publicado a finales de la década de 1980 (Garcia-Ramon y Caballé, 1998), una joven colega y yo misma detectamos unas formas diferentes de la evolución de la geografía del género según los países y lo estudiamos a través del análisis de los artículos de setenta y cinco revistas geográficas publicadas en veintitrés países y en doce lenguas diferentes. Los diversos temas tratados se podían agrupar en cuatro modelos regionales de desarrollo de esta geografía. Las revistas anglófonas — que eran la mayoríase caracterizaban por un enorme peso de la teoría, por un alto nivel de abstracción y por tratar temas como la sexualidad, la masculinidad y la posicionalidad, prácticamente inexistentes en los demás modelos en aquellos momentos. En las revistas francófonas y del área mediterránea, abundaban los estados de la cuestión sobre las novedades de la geografía feminista en el mundo anglófono - lo que demuestra una cierta dependencia metodológica de ésta-, pero dichas publicaciones también se distinguían por el peso de los temas del mundo rural, con lo cual acusaban la gran tradición de la geografía rural en estos países. En el tercer modelo regional — países escandinavos y centroeuropeos-, eran mayoritarios los temas de ocupación y mercado de trabajo, con lo cual acusaban la importante participación de la mujer en el mercado laboral y el interés por el estado de bienestar, en particular en los países escandinavos. Y, finalmente, en el cuarto modelo - el de los países del Tercer Mundo, y sobre todo de América Latina-, predominaban los temas urbanos - en particular la participación de las mujeres en los movimientos de base- - y reflejaban la difícil realidad de la vida política y social de las ciudades de América Latina durante el periodo en que se agravaron los problemas del endeudamiento exterior. Así mismo, en este modelo, también los temas rurales tenían un peso muy significativo (mayor que en las revistas francófonas y mediterráneas), lo cual indicaba la fuerte presencia del sector primario en el Tercer Mundo de esas décadas.

Hace poco, yo misma, junto con una colega griega y otra danesa, publicamos un editorial en Gender Place and Culture sobre el tema de la hegemonía angloamericana y, bajo esta perspectiva, analizamos el contenido de la revista desde su origen en 1994 hasta 2005 (es decir, doce años) (Garcia-Ramon et al., 2006). Estudiamos la procedencia de los autores, la de las referencias bibliográficas incluidas en los artículos, así como la de los miembros del Consejo Editorial de la revista.

Observamos que, de un total de 242 autores, solo 19 no pertenecían a universidades o centros de investigación angloamericanos, es decir, un 7,3\%. La autoría de EEUU y de Gran Bretańa representaba (por partes casi iguales) el $64 \%$ del total y la del Canadá anglófono, un 19\%. Se ha de constatar que el porcentaje de autoría de fuera del dominio anglófono no se incrementaba, sino que sucedía lo contrario. Agrupando los datos en periodos de tres años, el primer trienio arrojaba un $6 \%$ de contribuciones; el segundo, un 17\%; el 
tercero, un $10 \%$, y el último periodo volvía otra vez al $6 \%$. Al analizar la lengua de los libros reseńados, vimos que, de un total de 320, tan sólo 7 no estaban escritos en inglés, es decir, un 2,19\%, y en relación con los autores de las reseñas, tan sólo el 5\% no eran anglosajones (provenían de siete países diferentes: tres europeos, tres asiáticos y uno de América Latina).

En las bibliografías de los artículos, observamos que las referencias eran masivamente en inglés y llegaban al 95\% del total. El 5\% restante se repartía entre el francés, el alemán y el español. Y cabe señalar que, en tres de los números examinados, no había ni una sola referencia que no fuera en inglés. Casi la totalidad de la bibliografía no inglesa la citaban sobre todo los autores de fuera del ámbito anglosajón, aunque algunos autores anglófonos incluían referencias bibliográficas no inglesas cuando escribían sobre estudios de casos de fuera del ámbito anglosajón.

También analizamos la composición del Consejo Editorial y observamos que representaba muy poco las diferentes tradiciones de geografías feministas en el mundo. Si bien es cierto que, al principio de la revista (1994), cuatro de los diecinueve miembros del Consejo Editorial eran de fuera del ámbito angloamericano (de Francia, Grecia, Alemania y Canadá francés), muy pronto (al año siguiente) fueron desplazados al Consejo Asesor (que es mucho más amplio y con mucha menos incidencia). En el año 2000, se incluyó en el Consejo Editorial una nueva persona de fuera del ámbito anglosajón (de Singapur), pero, en 2003, los tres miembros no anglosajones del Consejo Asesor fueron dados de baja. No obstante, cabe señalar que, desde 1995, una de las editoras no pertenece al ámbito anglosajón (de Singapur). Además, cabe recordar que fue una de las editoras de la revista, Linda Peake, la que nos animó a realizar este tipo de estudio sobre la revista.

Pero parece que los cambios son difíciles o sobre todo lentos y se tardará en ver los resultados. Desde 2006, la composición del Consejo Editorial de la revista y las editoras no ha registrado grandes cambios en este sentido, si bien se ha de destacar que la editora jefe es de Singapur (era ya editora desde 1995) y una de las nuevas editoras es de la periferia del mundo anglosajón (Nueva Zelanda). También es cierto que, observando la lista de nombres de los censores anónimos de los dos años estudiados (218 nombres en 2009 y 205 en 2010), la gran mayoría pertenece al mundo anglófono, pero aparece también un número significativo de censores que no son originarios de la esfera anglosajona.

Recientemente, he puesto al día el análisis numérico que se publicó en 2006 para todos los números de la revista de 2009 y 2010 (doce números en total, una cifra igual a la de los trienios analizados anteriormente). Es interesante seńalar que, en este nuevo análisis, se observan luces y sombras. Por ejemplo: el número de autores de fuera del ámbito anglosajón ha aumentado ligeramente hasta un 13\%, destaca el papel de Turquía y algunos países europeos (también se ha incrementado el papel de países anglófonos "periféricos» como Nueva Zelanda y Australia). Es evidente que se trata del resultado de una cierta política editorial que aplaudimos, pero el número de referencias en 
inglés en los artículos sobrepasa el 95\% anterior (las no anglosajonas constituyen un 3,2\%), lo que es un reflejo de la creciente hegemonía de la literatura anglófona en este enfoque. Asimismo, en los dos años, observamos que, del total de treinta y tres reseñas de libro, tan sólo hay uno que no se ha escrito en inglés (en catalán). Pero, entre los 64 autores de las reseñas, el porcentaje de autores no anglosajones ha aumentado sensiblemente a un $14 \%$ y ello también se debe indiscutiblemente a la política editorial de la revista. Asimismo, cabe recordar que, desde hace un par de años, se publican los resúmenes en chino y es de esperar que esta política de cierta apertura de la revista consiga sus frutos en el próximo futuro.

\section{Conocimientos «situados» en geografia feminista}

Jan Monk (1995) afirmaba que el lugar es una categoría fundamental en el desarrollo de la geografía del género y que, por consiguiente, las tradiciones geográficas de los países pesaban mucho. El lugar, pues, importa en la producción de conocimientos situados y el predominio de la geografía feminista anglófona se ha reflejado en la selección de temas estudiados. Y así lo constatamos en la editorial de Gender, Place and Culture a la que me he referido anteriormente, pues es evidente que el peso de las elaboraciones teóricas es muy importante en esta revista, surgida en unos momentos en que la geografía anglosajona se caracterizaba por la crítica a las "grandes narrativas" y la fuerte irrupción del "giro cultural», hechos que se reflejan en su contenido. Así pues, abundan artículos sobre temáticas relacionadas con la identidad, la diferencia, la raza, la sexualidad, la "performatividad» de las masculinidades y las feminidades, las identidades queer y los discursos postcoloniales, el (trans) nacionalismo y la ciudadanía. En cambio, tienen poca presencia temas corrientes en la geografía del género en España y América Latina, como, por ejemplo: empleo, globalización y reestructuración económica, violencia, activismo y movimientos urbanos y rurales desde la base. Es cierto, sin embargo, que, en los últimos años, los temas de sexualidad han penetrado en estas otras geografías feministas, en particular en la brasileña.

En un seminario internacional que organizamos en Barcelona en 2006, titulado "Geografías del género en el mundo: cuestionando la hegemonía anglosajona" (BELGEO, 2007; Documents d'Anàlisi Geogràfica, 2007), también constatamos que la temática de la geografía feminista se veía muy influenciada por las diversas tradiciones geográficas de los diferentes países (en el seminario estaban representados catorce de ellos). La geografía británica era la más proclive a elaborar teorías de tipo general y, en cambio, en los países del África subsahariana, las investigadoras feministas buscaban temas relacionados con las culturas locales (por ejemplo: diferencias en la religión, la etnicidad, la edad, etc.), y estaban preocupadas porque existía un fuerte sesgo "occidental» en las teorías sobre el desarrollo que habían penetrado en los estudios feministas locales. En cambio, la geografía del género de América Latina se orientaba mucho más hacia los análisis de clase social, 
de la fuerza de trabajo de las mujeres y temas de activismo, sobre todo en las zonas urbanas (Lan y Silva, 2007). En el caso de España, la geografía feminista en los años ochenta y principios de los noventa se dedicó a estudiar temas rurales, siguiendo, por una parte, la tradición ruralista de la geografía española y, por otra, reflejando la fuerte crisis en la agricultura española al entrar en el Mercado Común. Como la mayoría de las participantes en el seminario no pertenecían al mundo anglosajón, pocas referencias se hicieron a los temas en boga en dicho ámbito, como, por ejemplo: el cuerpo, la sexualidad y la reflexividad y el rol de los investigadores (Garcia-Ramon y Monk, 2007).

\section{Mirando al futuro y produciendo conocimientos «situados»}

Algunas voces autorizadas de la geografía angloamericana crítica también se han sumado a esta necesidad de desestabilizar la hegemonía angloamericana y del inglés en nuestra disciplina (Kitchin, 2005). Es necesario, pues, abrir la geografia a una mayor pluralidad de voces, a formas diferentes de "hacer» geografía y a métodos alternativos de evaluar el trabajo académico en geografía. Y para ello deberíamos buscar foros alternativos para la discusión internacional en esta disciplina. No creo que sea solución cerrarnos en nuestra propia tradición y en nuestra escuela nacional, aunque, muchas veces, estemos tentados de hacerlo. El parroquialismo es peligroso y significa caer en el mismo tipo de errores que estamos criticando hoy en el mundo de habla inglesa. No es este el lugar ni el momento para enumerar y discutir las posibles soluciones concretas, pero sí me gustaría simplemente indicar algunas estrategias posibles.

Un posible camino es fomentar el plurilinguismo en la formación de los geógrafos. En efecto, tenemos que erradicar el monolingüismo de la geografía (ya sé que la idea es algo utópica, pero no por ello la he de dejar de mencionar). Los geógrafos académicos tendrían que poder leer más de una lengua extranjera. Los geógrafos que no son de habla inglesa han hecho un gran esfuerzo en este sentido en las últimas décadas, y pocos de ellos son monolingües, pero los geógrafos angloamericanos no parecen haber hecho ningún esfuerzo de similares características.

Tenemos que aceptar que, hoy en día, el inglés hace funciones de lingua franca y que esto por ahora es irreversible. Es verdad, pero otra forma de romper el imperio del monolingüismo anglófono es batallar en todos los foros internacionales para conseguir que otras lenguas (aparte del inglés) tengan el estatus de idioma de trabajo y que sean aceptadas en las publicaciones de los eventos realmente internacionales. En este sentido, la Unión Geográfica Internacional es la plataforma más universal de que disponemos en nuestra disciplina y, por lo tanto, se debería potenciar.

Una tercera posibilidad es trabajar para conseguir publicaciones verdaderamente internacionales en las que las "Otras» voces puedan ser escuchadas aunque también sometidas al mismo tipo de rigor metodológico (y esto es 
importante). Por un lado, ello podría implementarse abriendo las revistas académicas «internacionales» a otras lenguas, además del inglés (o, alternativamente, facilitar a los académicos no anglófonos la revisión de sus artículos en la lengua propia); por otro lado (y quiero subrayar esto en particular), se debería abrir el sistema de evaluación, ampliando la red de evaluadores anónimos y escogiéndolos de un mayor número de países. Algunas revistas anglosajonas (como, por ejemplo: Social and Cultural Geography, Antipode, $A C M E$ ) estan llevando a cabo un esfuerzo en este sentido, publicando (y no sólo en inglés) contribuciones de distintas tradiciones académicas, así como comentarios extensos sobre libros destacados escritos en lenguas no inglesas. Pero quiero insistir en que también debemos intentar que revistas de fuera del mundo anglosajón se sitúen, por su calidad y su rigor metodológico, en un puesto destacado a nivel internacional y lleguen a tener índices de calidad reconocidos a nivel internacional.

En síntesis, no quiero sugerir que todos nosotros tengamos que hablar muchas lenguas, sólo que, de forma colectiva, tenemos que intentar dar una cobertura más adecuada a lo que se hace en la geografía fuera de nuestro propio entorno. Ni aquí ni en la geografía de habla inglesa debemos encerrarnos en nuestro mundo, esto lo tengo muy claro. Se trata de un camino largo y difícil, pero todo intento de favorecer un pluralismo disciplinario vale el esfuerzo a fin de hacer posible los intercambios reales que sólo pueden llevarse a cabo desde la pluralidad y la hibridación.

\section{Referencias bibliográficas}

Aalbers, Manuel B. y Rossi, Ugo (2007). «A coming community: Young geographers coping with multi-tier academic spaces across Europe». Social and Cultural Geography, 8, 283-302.

- (2009). "Anglo-American/Anglophone Hegemony». En: Kitchin, Rob y Thrift, Nigel (eds.). International Encyclopedia of Human Geography. Londres: Elsevier, 116-121.

Albet, Abel y Benach, Núria (Margarita de la Vez) (2005). «Conocimientos situados: reflexión sobre las geografías de la Geografía (Crónica de una viaje al 100 Congreso de la Association of American Geographers)». Documents d'Anàlisi Geogràfica, 45, 131-138.

BELGEO: Revue Belge de Géographie (2007). Número monográfico sobre «Feminist Geographies Around the World».

Belina, Berdn; Best, Ulrich y Naumann, Mathias (2009). "Critical geography in Germany: From exclusion to inclusion». Social Geography, 4, 47-58.

Berg, Lawrence y KeARn, Robin (1998). "America unlimited». Environment and Planning D, 16, 128-132.

Best, Ulrich (2009). "The invented periphery: Constructing Europe in debates about "Anglo hegemony" in geography". Social Geography, 4, 83-91.

BRAUN, Bruce (2003). «Introduction: tracking the power geometries of international critical geography». Environmental and Planning D, 21, 131-133.

Castells, Manuel (1972). La question urbaine. París: François Maspero. Traducción 
inglesa: The Urban Question: a Marxist Approach. Londres: Edgard Arnold, 1977.

Critical geographical forum archives (1998). Comentarios escritos en Internet.

Derudder, Ben (2011). "Some reflections on the "problematic" dominance of "Web of Science" journals in academic human geography". Area, 43 (1), 110-112.

Documents d'Anàlisi Geogràfica (2007). Número monográfico «Una mirada internacional a la geografia i el gènere», 49.

Garcia-Ramon, Maria Dolors (2003). «Globalization and internacional geography: the questions of languages and scholarly traditions». Progress in Human Geography, 27 (1), 1-5.

- (2004). «The spaces of critical geography: An introduction». Geoforum, 35, 523524.

Garcia-Ramon, Maria Dolors y Caballé, Alba (1998). «Situating gender geographies: a Biblioometrical analysis». Tijdschrift voor Economische en Sociale Geografie, 89 (2), 210-216.

Garcia-Ramon, Maria Dolors y Monk, Jan (2007). "Gender and geography: worlds views and practices». BELGEO: Revue Belge de Géographie, 3, 247-259.

Garcia-Ramon, Maria Dolors; Simonsen, Kirsten y Vaiou, Dina (2006). "Does Anglophone hegemony permeate Gender, Place and Culture?». Gender, Place and Culture, 13 (1), 1-5.

Gutiérrez, Javier y López-Nieva, Pedro (2001). «Are internacional journals of human geography really internacional?». Progress in Human Geography, 25, 53-69.

Hancock, Claire (1999). «Du centre et de la péripherie en gèographie culturelle». Géographies et Cultures, 32, 129-132.

Haraway, Donna (1995). «Conocimientos situados: La cuestión científica en el feminismo y el privilegio de la perspectiva parcial». En: Haraway, H. Ciencia, cyborgs y mujeres: La reinvención de la naturaleza. Valencia: Cátedra, 313-346.

Kitchin, Rob (2003). "Cuestionando y desestabilizando la hegemonía angloamericana y del inglés en geografía». Documents d'Anàlisi Geogràfica, 42, 17-36.

- (2005). «Disrupting and destabilizing Anglo-American English-language hegemony in geography». Social and Cultural Geography, 6, 1-15.

Lan, Diana y Silva, Susana (2007). «Estudios de geografia del género en América Latina: Un estado de la cuestión a partir de los casos de Brasil y de Argentina». Documents d'Anàlisi Geogràfica, 49 (3), 99-118.

Meeus, Bruno; Schuermans, Nick y De Maesschalck, Filip (2011). "Is there a World beyond academia geography? A reply to Ben Derudder». Area, 43 (1), 113-114.

Minca, Claudio (2003). "Critical Peripheries». Environment and Planning D, 18, 285-289.

Monk, Jan (1995). «El lloc compta: Perspectives internacionals comparades sobre la geografia feminista». Documents d'Anàlisi Geogràfica, 26, 254-259.

PAAsI, Ansii (2005). "Globalisation, academic capitalism, and the uneven geographies of international journal publishing spaces». Environment and Planning $A$, 37 (5), 769-789.

PeAke, Linda (2011). «In, out and unspeakably about: taking social geography beyond and Anglo-American positionality». Social and Cultural Geography, $12(7), 757-773$. 
Rodríguez-Pose, Andrés (2004). «On English as a vehicle to preserve geographical diversity». Progress in Human Geography, 28, 1-4.

- (2006). "Is there an "Anglo-American" domination in human geography? And, is it bad?». Environment and Planning A, 22, 647-651.

Samers, Michael y Sidaway, James D. (2000). «Exclusions, inclusions, and occlusions in "Anglo-American geography": Reflections on Minca's "Venetian Geographical Praxis"». Environmental and Planning D, 18, 663-666.

Short, John; Boniche, Armando; Kim, Yeong y Li Li, Patrick (2001). «Cultural globalization, global English and geography journals». The Professional Geographer, 53, 1-11.

Timar, Judit (2004). «More than "Anglo-American", it is "Western": Hegemony in geography from a Hungarian perspective». Geoforum, 35 (5), 533-538.

Vaiou, Dina (2004). "The contested and negotiated dominance of Anglophone geography in Greece». Geoforum, 35, 529-531.

Women and Geography Study Group (1997). Feminist Geographies: Exploration in diversity and difference. Londres: Longman. 